\title{
Modelado cinemático y dinámico de un manipulador antropomórfico de cuatro grados de libertad
}

\author{
Ibarra-Ontiveros, Eddy G. ${ }^{a}$, Enríquez-Rocha, Manuel A. ${ }^{a}$, Galván-Guerra, Rosalba ${ }^{\mathrm{b}}$, Maya-Rodríguez, Mario C. ${ }^{\mathrm{c}}$, Lozano \\ Hernández, Yair ${ }^{\mathrm{a}, 1, *}$ \\ ${ }^{a}$ Escuela Superior de Ingeniería Mecanica y Eléctrica unidad Zacatenco, Instituto Politécnico Nacional, CDMX, México. \\ ${ }^{b}$ Unidad Profesional Interdisciplinaria de Ingeniería campus Hidalgo, Instituto Politécnico Nacional, Hidalgo, México. \\ ${ }^{c}$ Centro de Investigación y de Estudios Avanzados del Instituto Politécnico Nacional unidad Zacatenco, CDMX, México.
}

\section{Resumen}

En el presente trabajo se desarrolla el modelo cinemático y dinámico de un robot manipulador tipo antropomórfico de cuatro grados de libertad mediante la convención de Denavit-Hartenberg y Euler-Lagrange respectivamente. Se hace uso del modelo cinemático para la construcción del espacio de trabajo del manipulador. La estimación de los parámetros físicos del manipulador se hace mediante el uso de un software CAD-CAE. Posteriormente se valida el modelo dinámico del manipulador mediante la comparación de sus resultados con los obtenidos a través de una herramienta computacional que emplea el método de modelado de Newton-Euler.

Palabras Clave: Robot manipulador, Robot antropomórfico, Modelo cinemático, Modelo dinámico, Euler-Lagrange.

\section{Introducción}

Los robots manipuladores son de gran utilidad para el ser humano y su uso se ha extendido en diferentes industrias (automotriz, de alimentos, metalúrgica, etc.) gracias a su capacidad para realizar tareas que resultarían muy cansadas o peligrosas para las personas,como por ejemplo, manipulación de objetos pesados o separación y clasificación de alimentos (Shepherd and Buchstab, 2014; Meike et al., 2013; Chua et al., 2003; Markert and Merk, 2009). Formalmente un robot se define como un manipulador multifuncional reprogramable diseñado para mover materiales, piezas, herramientas o dispositivos especializados a través de movimientos variables programados para la realización de una variedad de tareas (Spong and Vidyasagar, 2008).

El estudio de los manipuladores se centra principalmente en su cinemática y dinámica, planificación de trayectorias y control de movimiento. La cinemática estudia el movimiento del manipulador en el espacio de trabajo en función de las posiciones articulares del mismo, sin considerar las fuerzas que producen dicho movimiento. El modelo dinámico considera las fuerzas y pares que convergen en el movimiento, tomando en cuenta

\footnotetext{
*Autor en correspondencia. ylozanoh@ipn.mx

Correos electrónicos: eibarrao1300@alumno.ipn.mx (Ibarra-Ontiveros, Eddy G.), m. alejandro.enriquez.r@gmail.com (Enríquez-Rocha, Manuel A.), rgalvang@ipn.mx (Galván-Guerra, Rosalba), mario.c.maya.r@hotmail.com (Maya-Rodríguez, Mario C.), ylozanoh@ipn.mx (Lozano Hernández, Yair )
}

los efectos inerciales, pares gravitaciones, fuerzas centrípetas y de coriolis presentes en la dinámica acoplada de los eslabones (Spong and Vidyasagar, 2008; Kelly et al., 2006; Murray, 2017; Corke, 2007). El estudio del movimiento del manipulador en su espacio de trabajo es indispensable para la correcta planificación de las trayectorias deseadas, por lo tanto; es necesario obtener el modelo cinemático del manipulador (ver (RamírezGordillo, 2010; Merchán-Cruz, 2000; Flores, 2006)). Por otra parte, el diseño de algoritmos de control de movimiento para el manipulador requiere, en su mayoría, del conocimiento del modelo dinámico, debido a que el algoritmo de control es responsable de calcular los pares que se deben aplicar a las articulaciones del y compensar la dinámica acoplada de los eslabones, para así realizar un adecuado seguimiento de las trayectorias deseadas (Arimoto, 1986; Antonio, 2012).

En este artículo se describe el procedimiento para obtener el modelo cinemático de un robot manipulador de cuatro grados de libertad mediante el enfoque de Denavit-Hartenberg, de igual manera; se obtiene el espacio de trabajo y se calcula el modelo dinámico del mismo a través de la ecuación de EulerLagrange. En trabajos previos el análisis de manipuladores se centra principalmente en configuraciones estandarizadas tales como el manipulador SCARA (Gautier, 1997), Stanford, etc. o bien en robots planares (Flores, 2006). Por lo anterior, en el presente trabajo se hace el análisis de un manipulador con una configuración que no suele estudiarse con tanta frecuencia y se incluyen las repercusiones que esto tiene en el desarrollo de su 
modelo dinámico.

Dada la relevancia de los modelos cinemático y dinámico del manipulador, los esfuerzos de este trabajo se centran en la validación de los mismos, esto mediante simulación numérica y comparación de los resultados de los modelos calculados, con los obtenidos al usar una herramienta computacional para el cálculo de dichos modelos.

El presente trabajo se organiza de la siguiente manera: La sección 2 describe las consideraciones tomadas sobre el manipulador, así como el objetivo de este trabajo. En la sección 3 se presenta el procedimiento general para la obtención del modelo cinemático y dinámico, al igual que los resultados obtenidos. De igual manera se incluye el cálculo del espacio de trabajo. La sección 4 detalla el proceso de identificación de los parámetros físicos del manipulador por medio de un software CAD-CAE. Del mismo modo, se describe la técnica usada para simular el modelo dinámico obtenido y así estudiar la evolución de las posiciones articulares ante ciertos pares de entrada. En la sección 5 se contrastan los resultados del modelo desarrollado mediante la formulación de Euler-Lagrange con los obtenidos a través del uso de una herramienta computacional que emplea el método de Newton-Euler. La sección 6 contiene las conclusiones derivadas de la realización de este trabajo.

\section{Planteamiento del problema}

El manipulador analizado es de tipo antropomórfico y cuenta con cuatro grados de libertad (DOF, por sus siglas en inglés), lo cual indica que las juntas son de tipo rotacionales y corresponden a la articulación de cintura, hombro, codo y muñeca, considerando un efector final de tipo pinza al final del cuarto eslabón. La configuración del manipulador se muestra en la Figura 1, en la cual se observa que el manipulador posee un actuador en el inicio del efector final, el cual tiene un eje de rotación perpendicular a los ejes de rotación de los tres actuadores anteriores, en este caso dicho actuador se limita a mantener una posición constante, por lo que no se considera como un grado de libertad, del mismo modo; el actuador responsable de accionar el efector final tampoco se toma como un DOF, puesto que su acción no modifica la posición u orientación del efector final.

El principal fin práctico de un manipulador es el seguimiento de una trayectoria deseada en su espacio de trabajo, con el objetivo de realizar una serie de tareas determinadas. La planificación de estas tareas conlleva un estudio sobre los límites y alcances del movimiento del manipulador. Por otra par-

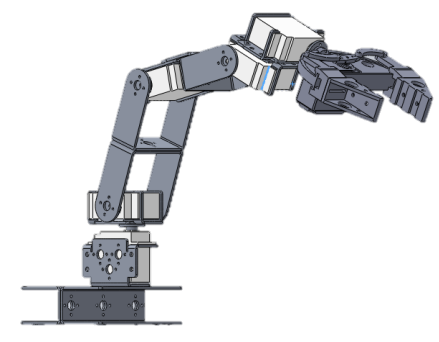

Figura 1: Modelo virtual del manipulador en el software CAD te, el desempeño en la ejecución de dichas tareas depende de la velocidad y precisión con la que el manipulador sea capaz de efectuar sus movimientos. Tomando esto en consideración, queda clara la importancia de contar con el modelo cinemático y dinámico del manipulador para la planificación de trayectorias y diseño de algoritmos de control.

\section{Modelado Cinemático y Dinámico}

\subsection{Modelado Cinemático}

Para obtener el modelo cinemático del manipulador se realiza la asignación de los sistemas de referencia para cada eslabón. Con base en la convención Denavit-Hartenberg (en adelante DH) un sistema de coordenadas fijo es asignado a la base del manipulador como sistema cero, posteriormente se asignan los sistemas de referencia en cada eslabón, cumpliendo con que el eje $Z$ de cada sistema sea perpendicular al plano en el que se realiza la rotación y satisfaciendo los parámetros DH1 y DH2 Spong and Vidyasagar (2008). Como resultado se obtiene el esquema de la Figura 2, donde cabe resaltar que los sistemas de referencia correspondientes a cada eslabón se encuentran al final de los mismos.

- DH1: El eje $X_{1}$ sea perpendicular al eje $Z_{0}$

- DH2: El eje $X_{1}$ intersecta al eje $Z_{0}$

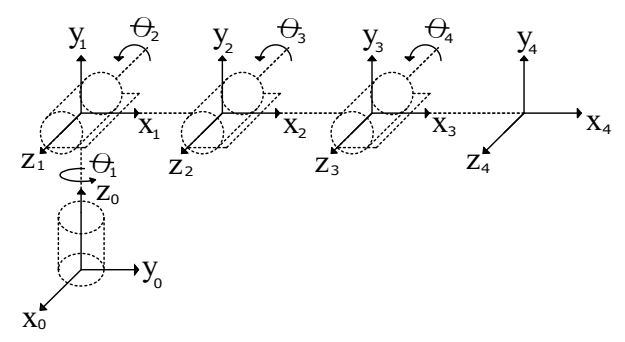

Figura 2: Representación de los marcos de referencia en el manipulador

Utilizando los sistemas de referencia propuestos, los parámetros de rotación y traslación se agrupan en la Tabla 1 de parámetros de $\mathrm{DH}$, donde los valores de $\theta_{i}$ son variables y el resto son constantes.

\begin{tabular}{|c|c|c|c|c|}
\hline Eslabon & $a_{i}$ & $\alpha_{i}$ & $d_{i}$ & $\theta_{i}$ \\
\hline 1 & 0 & $\frac{\pi}{2}$ & $\mathrm{~d} 1$ & $\theta_{1}$ \\
\hline 2 & $a_{2}$ & 0 & 0 & $\theta_{2}$ \\
\hline 3 & $a_{3}$ & 0 & 0 & $\theta_{3}$ \\
\hline 4 & $a_{4}$ & 0 & 0 & $\theta_{4}$ \\
\hline
\end{tabular}

Tabla 1: Parámetros de Denavit Hartenberg

Con base en estos parámetros se encuentran las matrices de transformación homogénea.

$$
\mathbf{A}_{1}=\left(\begin{array}{cccc}
c_{\theta_{1}} & 0 & s_{\theta_{1}} & 0 \\
s_{\theta_{1}} & 0 & -c_{\theta_{1}} & 0 \\
0 & 1 & 0 & d_{1} \\
0 & 0 & 0 & 1
\end{array}\right)
$$




$$
\begin{aligned}
\mathbf{A}_{2} & =\left(\begin{array}{cccr}
c_{\theta_{2}} & -s_{\theta_{2}} & 0 & a_{2} c_{\theta_{2}} \\
s_{\theta_{2}} & c_{\theta_{2}} & 0 & a_{2} s_{\theta_{2}} \\
0 & 0 & 1 & 0 \\
0 & 0 & 0 & 1
\end{array}\right) \\
\mathbf{A}_{3} & =\left(\begin{array}{cccr}
c_{\theta_{3}} & -s_{\theta_{3}} & 0 & a_{3} c_{\theta_{3}} \\
s_{\theta_{3}} & c_{\theta_{3}} & 0 & a_{3} s_{\theta_{3}} \\
0 & 0 & 1 & 0 \\
0 & 0 & 0 & 1
\end{array}\right) \\
\mathbf{A}_{4} & =\left(\begin{array}{cccr}
c_{\theta_{4}} & -s_{\theta_{4}} & 0 & a_{4} c_{\theta_{4}} \\
s_{\theta_{4}} & c_{\theta_{4}} & 0 & a_{4} s_{\theta_{4}} \\
0 & 0 & 1 & 0 \\
0 & 0 & 0 & 1
\end{array}\right)
\end{aligned}
$$

donde $c_{1}, s_{1}, c_{12}$ y $s_{12}$ son $\cos \theta_{1}, \sin \theta_{1}, \cos \left(\theta_{1}+\theta_{2}\right)$ y $\sin \left(\theta_{1}+\theta_{2}\right)$ respectivamente. Las ecuaciones (1), (2), (3) y (4) describen el movimiento del eslabón producido por su articulación, para el movimiento del efector final en función de los valores de las articulaciones se calcula la matriz de transformación homogénea.

$$
\mathbf{H}_{\mathbf{4}}^{\mathbf{0}}=\mathbf{A}_{\mathbf{1}} \mathbf{A}_{2} \mathbf{A}_{\mathbf{3}} \mathbf{A}_{4}=\left(\begin{array}{cccc}
H_{11} & H_{12} & H_{13} & H_{14} \\
H_{21} & H_{22} & H_{23} & H_{24} \\
H_{31} & H_{32} & H_{33} & H_{34} \\
H_{41} & H_{42} & H_{43} & H_{44}
\end{array}\right)
$$

$$
\begin{aligned}
H_{11} & =c_{1} c_{2} c_{3} c_{4}-s_{2} s_{3} c_{1} c_{4}-c_{1} c_{2} s_{3} s_{4}-s_{2} s_{4} c_{1} c_{3} \\
H_{12} & =-c_{1} c_{2} c_{3} s_{4}+s_{2} s_{3} s_{4} c_{1}-c_{1} c_{2} c_{4} s_{3}-s_{2} c_{1} c_{3} c_{4} \\
H_{13} & =s_{1} \\
H_{14} & =a_{4} c_{1} c_{2} c_{3} c_{4}-a_{4} s_{2} s_{3} c_{1} c_{4}-a_{4} c_{1} c_{2} s_{3} s_{4}-a_{4} s_{2} s_{4} c_{1} c_{3} \\
& +a_{3} c_{1} c_{2} c_{3}-a_{3} s_{2} s_{3} c_{1}+a_{2} c_{1} c_{2} \\
H_{21} & =s_{1} c_{2} c_{3} c_{4}-s_{1} s_{2} s_{3} c_{4}-s_{1} s_{3} s_{4} c_{2}-s_{1} s_{2} s_{4} c_{3} \\
H_{22} & =-s_{1} s_{4} c_{2} c_{3}+s_{1} s_{2} s_{4} s_{3}-s_{1} s_{3} c_{2} c_{4}-s_{1} s_{2} c_{3} c_{4} \\
H_{23} & =-c_{1} \\
H_{24} & =a_{4} s_{1} c_{2} c_{3} c_{4}-a_{4} s_{1} s_{2} s_{3} c_{4}-a_{4} s_{1} s_{3} s_{4} c_{2}-a_{4} s_{1} s_{2} s_{4} c_{3} \\
& +a_{3} c_{2} c_{3} s_{1}-a_{3} s_{1} s_{2} s_{3}+a_{2} c_{2} s_{1} \\
H_{31} & =s_{2} c_{3} c_{4}+c_{2} c_{4} s_{3}-s_{2} s_{3} s_{4}+c_{2} c_{3} s_{4} \\
H_{32} & =-s_{2} s_{4} c_{3}-c_{2} s_{3} s_{4}-s_{2} s_{3} c_{4}+c_{2} c_{3} c_{4} \\
H_{33} & =0 \\
H_{34} & =a_{4} s_{2} c_{3} c_{4}+a_{4} c_{2} c_{4} s_{3}-a_{4} s_{2} s_{3} s_{4}+a_{4} c_{2} c_{3} s_{4}+a_{3} c_{3} s_{2} \\
& +a_{3} s_{3} c_{2}+a_{2} s_{2}+d_{1} \\
H_{41} & =0 \\
H_{41} & =0 \\
H_{41} & =0 \\
H_{41} & =1
\end{aligned}
$$

De este modo, haciendo uso de (5) se determina la posición y orientación del efector final en función de los valores de las articulaciones, la cual se utiliza en la obtención del modelo dinámico.

\subsection{Espacio de trabajo}

El espacio de trabajo se define como el área de barrido total del manipulador, limitado por el tipo y cantidad de juntas Spong and Vidyasagar (2008). Para calcular el espacio de trabajo alcanzable, se genera un código en matlab, definiendo los movimientos de cada eslabón en arreglos de cincuenta elementos para las posiciones $q$, se define la matriz de transformación homogénea y se crea un algoritmo que calcula los valores de dicha matriz para cada movimiento del manipulador, almacenando en vectores las coordenadas de la posición $[x, y, z]$ del efector final, conteniendo así todos los puntos del espacio de trabajo en coordenadas cartesianas (ver Figura 3).

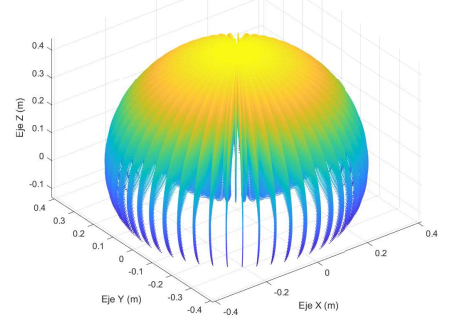

Figura 3: Espacio de trabajo del manipulador

\subsection{Modelado Dinámico}

La descripción del movimiento del sistema se realiza en función de la fuerza a la que está sometido. Estas fuerzas se pueden estudiar con base a la energía total contenida en el sistema, para esto se hace uso del Lagrangiano

$$
L(q, \dot{q})=K(q, \dot{q})-U(q)
$$

donde $q=\left[q_{1}, q_{2}, \ldots, q_{n}\right]^{T}, \dot{q}=\left[\dot{q}_{1}, \dot{q}_{2}, \ldots, \dot{q}_{n}\right]^{T}$ corresponde a las posiciones y velocidades articulares, $K(q, \dot{q})$ es la energía cinética y $U(q)$ la energía potencial. La energía cinética total $K_{T}$ está definida por la suma de la energía cinética traslacional $K_{t}$ y la energía cinética rotacional $K_{r}$. Por otro lado, $U$ tiene una dependencia directa de la altura a la que se encuentra el objeto (Goldstein et al., 2002).

$$
\begin{gathered}
K_{T}=K_{t}+K_{r}=\frac{1}{2} m v^{2}+\frac{1}{2} I w^{2} \\
U=h g m
\end{gathered}
$$

donde $v$ es velocidad lineal, $w$ velocidad angular, $m$ la masa, $I$ el momento de inercia, $h$ la altura y $g$ la constante de aceleración gravitacional.

Para el caso del manipulador, la energía cinética total está dada por la suma de las energías cinéticas de cada eslabón, de la misma manera, la energía potencial total del manipulador es la suma de las energías potenciales (Eqs. 9 - 10).

$$
K_{T M}=K_{T 1}+K_{T 2}+K_{T 3}+K_{T 4}
$$




$$
U_{T M}=U_{1}+U_{2}+U_{3}+U_{4}
$$

Al tratarse de cuerpos rígidos, la energía cinética de cada eslabón se calcula en función del desplazamiento de su centro de masa y de las rotaciones presentes en su sistema de referencia, todo con respecto al sistema de referencia fijo. Igualmente, la energía potencial se calcula en función de la altura del centro de masa de cada eslabón con respecto al sistema de referencia fijo (Goldstein et al., 2002).

Para el cálculo de la velocidad lineal de los centros de masa de cada eslabón con respecto a la base del manipulador se toma como referencia la Figura 4, donde las distancias de los centros de masa se denotan por $l_{c i}$.

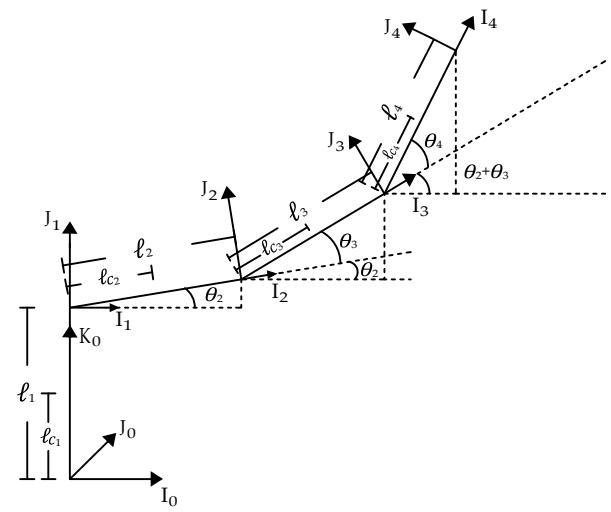

Figura 4: Centros de masa del manipulador

El vector de posición que describe el desplazamiento del centro de masa del eslabón en el sistema de referencia base, este esta en función de la longitud de cada eslabón $l_{i}$ y su movimiento angular $q_{i}$ así las posiciones de $x, y$ y $z$ se expresan de la forma

$$
\mathbf{p}_{\mathbf{I c}_{\mathbf{i}}}=\left[\begin{array}{c}
x\left(l_{i}, q_{i}\right) \\
y\left(l_{i}, q_{i}\right) \\
z\left(l_{i}, q_{i}\right)
\end{array}\right]
$$

La velocidad del eslabón respectivo está definida como

$$
\mathbf{v}_{\mathbf{l} \mathbf{c}_{\mathbf{i}}}=\left[\begin{array}{l}
\dot{x}\left(l_{i}, q_{i}, \dot{q}_{i}\right) \\
\dot{y}\left(l_{i}, q_{i}, \dot{q}_{i}\right) \\
\dot{z}\left(l_{i}, q_{i}, \dot{q}_{i}\right)
\end{array}\right]
$$

Así, la energía cinética traslacional de cada eslabón es

$$
K_{t_{i}}=\frac{1}{2} m_{i} v_{l c_{i}}^{T} v_{l c_{i}}
$$

donde $v_{l c_{i}}$ es velocidad lineal del centro de masa del eslabón en el sistema de referencia base y $m_{i}$ la masa del eslabón.

La energía cinética rotacional utiliza la velocidad angular del eslabón con el tensor de inercia asociado a dicho eslabón, ambos expresados en el sistema de referencia fijo

$$
K_{r_{i}}=\frac{1}{2}{ }^{0} w_{i}^{T 0} I_{i}^{0} w_{i}
$$

donde ${ }^{0} w_{i}$ es la velocidad angular e ${ }^{0} I_{i}$ el tensor de inercia del eslabón en el sistema de referencia base.

El tensor ${ }^{0} I_{i}$ varía con el movimiento del eslabón, pues este cambia su posición con respecto al sistema de referencia de la base. Bajo la suposición de un tensor de inercia constante, dado en el sistema de referencia fijo del propio eslabón, se aplica una transformación rígida hacia el sistema de referencia de la base. Para dicha transformación se definen dos momentos angulares dados en dos sistemas de referencia distintos

$$
\begin{aligned}
& { }^{0} h={ }^{0} I^{0} w \\
& { }^{i} h={ }^{i} I^{i} w
\end{aligned}
$$

donde ${ }^{0} w$ es la velocidad angular ${ }^{0} I$ el tensor de inercia, ambos dados en el sistema 0 ; mientras que ${ }^{i} w$ es la velocidad angular $\mathrm{e}^{i} I$ el tensor de inercia, ambos dados en el sistema $i$. Entonces, el cambio de referencia del momento angular ${ }^{i} h$ al sistema de referencia 0 está dado por

$$
{ }^{0} h=\left({ }^{0} R_{i}\right)\left({ }^{i} h\right)=\left({ }^{0} R_{i}\right)\left({ }^{i} I^{i} w\right)=\left({ }^{0} R_{i}\right)\left({ }^{i} I^{0} R_{i}^{T 0} w\right)
$$

Observe que el tensor de inercia ${ }^{i} I$ dado en el sistema 0 es:

$$
{ }^{0} I={ }^{0} R_{i}{ }^{i} I^{0} R_{i}^{T}
$$

donde las matrices de rotación $R_{i}$ se extraen directamente de las matrices de transformación homogénea definidas para cada eslabón. En consecuencia, la energía cinética rotacional de cada eslabón queda de la forma

$$
K_{r_{i}}=\frac{1}{2}{ }^{0} w_{i}^{T 0} R_{i} \tilde{I}_{i}^{0} R_{i}^{T 0} w_{i}
$$

${ }^{0} w_{i}$ es la velocidad angular del eslabón dada en el sistema de referencia base, $\tilde{I}_{i}$ el tensor de inercia del eslabón dado en el sistema de referencia fijo del mismo y ${ }^{0} R_{i}$ es la matriz de rotación del sistema de referencia del eslabón con respecto al sistema de referencia de la base.

La velocidad angular del eslabón en el sistema de referencia de la base del robot está definida por

$$
\mathbf{o}_{\mathbf{i}}=\left[\begin{array}{c}
i\left(q_{i}, \dot{q}_{i}\right) \\
j\left(q_{i}, \dot{q}_{i}\right) \\
k\left(q_{i}, \dot{q}_{i}\right)
\end{array}\right]
$$

La energía potencial de cada eslabón está relacionada con la altura de su respectivo centro de masa con respecto al sistema fijo, la altura está en función de las posiciones $q$ por lo que queda definida como

$$
h_{i}=z\left(l_{i}, q_{i}\right)
$$

La forma general de la energía potencial de los eslabones es

$$
U_{i}=h_{i} g m_{i}
$$

Así, se define el lagrangiano como

$$
\begin{aligned}
L(q, \dot{q}) & =K_{1}(q(t), \dot{q}(t))+K_{2}(q(t), \dot{q}(t))+K_{3}(q(t), \dot{q}(t)) \\
& +K_{4}(q(t), \dot{q}(t))-U_{1}(q)-U_{2}(q)-U_{3}(q)-U_{4}(q)
\end{aligned}
$$


La ecuación de Euler-Lagrange se define como:

$$
\frac{d}{d t}\left[\frac{\partial L(q, \dot{q})}{\partial \dot{q}_{i}}\right]-\frac{\partial L(q, \dot{q})}{\partial q_{i}}=\tau_{i}
$$

La cual relaciona el par aplicado a las articulaciones con una serie de operaciones derivativas aplicadas al lagrangiano, donde $\tau_{i}$ es el par aplicado a la i-ésima articulación (Spong and Vidyasagar, 2008).

La dinámica de cada eslabón se obtiene mediante (23). Esta ecuación describe la relación entre la posición, la velocidad y la aceleración del eslabón con el par aplicado a la articulación que mueve a dicho eslabón. Se obtienen 4 ecuaciones dinámicas, las cuales pueden ser agrupadas como

$$
\tau=\mathbf{M}(\mathbf{q}) \ddot{\mathbf{q}}+\mathbf{C}(\mathbf{q}, \dot{\mathbf{q}}) \dot{\mathbf{q}}+\mathbf{G}(\mathbf{q})
$$

donde $\ddot{\boldsymbol{q}}=\left[\ddot{q}_{1}, \ddot{q}_{2}, \ldots, \ddot{q}_{n}\right]^{T}$ son las aceleraciones, $\boldsymbol{\tau}=\left[\tau_{1}, \tau_{2}, \ldots\right.$, $\left.\tau_{n}\right]^{T}$ el vector de pares aplicados, $\mathbf{M}(\mathbf{q}) \in \mathbb{R}^{n x n}$ es la matriz de inercia, $\mathbf{C}(\mathbf{q}, \dot{\mathbf{q}}) \in \mathbb{R}^{n x n}$ la matriz centrípeta y de Coriolis y $\mathbf{G}(\mathbf{q}) \in \mathbb{R}^{n x 1}$ es el vector de pares gravitacionales. La definición de cada matriz se muestra en el Apéndice ${ }^{\sim}$ A.

\section{Identificación y simulación del prototipo}

El modelo dinámico del manipulador es esencial para el diseño de algoritmos de control, puesto que una gran cantidad de controladores basan su diseño en propiedades extraídas del modelo dinámico. Debido a esto, resulta crucial para el desempeño de los controladores que los parámetros físicos que describen al manipulador sean lo más cercanos posible a sus valores reales.

Retomando el manipulador de la sección 2, se hace uso del modelo virtual en el software CAE-CAD SolidWorks. Así, basándose en las dimensiones de la estructura y el material de construcción, se crea una aproximación que es capaz de simular condiciones físicas.

Utilizando el modelo virtual (ver Figura 1), se analizan las propiedades físicas de cada uno de los eslabones y se obtienen los parámetros que describen a este.

Sea el $i$-ésimo eslabón la parte de la estructura del manipulador que conecta la articulación $(i+1)$ con la $i$-ésima articulación, separando el modelo virtual del manipulador en 4 ensambles independiente correspondientes a los eslabones, cada eslabón del manipulador se analiza en SolidWorks.

A cada ensamble de eslabón se le asigna un nuevo sistema de referencia, de modo que este coincida con el sistema de referencia asignado en el cálculo del modelo cinemático. Las propiedades físicas calculadas mediante el uso del modelo virtual son la masa total, longitud total, centro de masa y el tensor de inercia de cada eslabón con respecto al nuevo sistema de referencia.

Otra característica física es la oposición al movimiento generada por la fricción viscosa en cada junta. Dado que la fricción resulta complicado de estimar, se considera solo la fricción presente en los servo motores acoplados a cada articulación, para esto se toma una identificación previa hecha para los motores usados en un manipulador de características similares al de este trabajo (Corke, 2017). Los resultados de la parametrización realizada se agrupan en el Apéndice ${ }^{\sim}$ B.

Utilizando los parámetros aproximados, la ecuación (24) es

$$
\tau=\mathbf{M}(\mathbf{q}) \ddot{\mathbf{q}}+\mathbf{C}(\mathbf{q}, \dot{\mathbf{q}}) \dot{\mathbf{q}}+\mathbf{G}(\mathbf{q})+\mathbf{B} \dot{\mathbf{q}}
$$

donde $\mathbf{B} \in \mathbb{R}^{n x 1}$ es el vector de constantes de fricción viscosa. De la 25 se obtiene el modelo dinámico directo

$$
\ddot{\mathbf{q}}=\mathbf{M}(\mathbf{q})^{-1}[\tau-(\mathbf{C}(\mathbf{q}, \dot{\mathbf{q}}) \dot{\mathbf{q}}+\mathbf{G}(\mathbf{q})+\mathbf{B} \dot{\mathbf{q}})]
$$

del cual se puede obtener el vector de posiciones articulares

$$
\mathbf{q}=\int_{0}^{t} \int_{0}^{t} \mathbf{M}(\mathbf{q})^{-1}[\tau-(\mathbf{C}(\mathbf{q}, \dot{\mathbf{q}}) \dot{\mathbf{q}}+\mathbf{G}(\mathbf{q})+\mathbf{B} \dot{\mathbf{q}})] \cdot d t \cdot d t
$$

Posterior a esto y usando los parámetros de la Tabla B.2, se procede a programar las ecuaciones dinámicas del manipulador en su forma matricial y se construye el diagrama de simulación analógica de la ecuación (27) (ver Figura 5). Así, se estudia la evolución de las posiciones ante los pares de entrada $\boldsymbol{\tau}_{0}=[0,1,0,3,0,1,0,2]$, partiendo de las posiciones iniciales $\mathbf{q}_{0}=[0,0,0,0]$. El tiempo de simulación se fija en 5 segundos, siendo este suficiente para obtener una referencia sobre la evolución de las posiciones articulares ante un par de entrada fijo. (ver Figura 6).

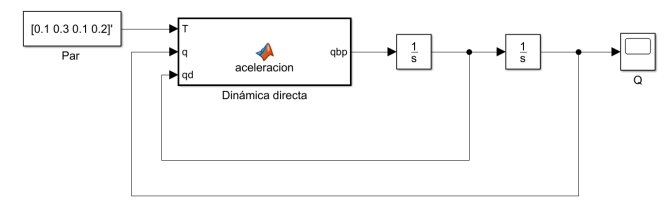

Figura 5: Diagrama de simulación analógica para el modelo cinemático directo

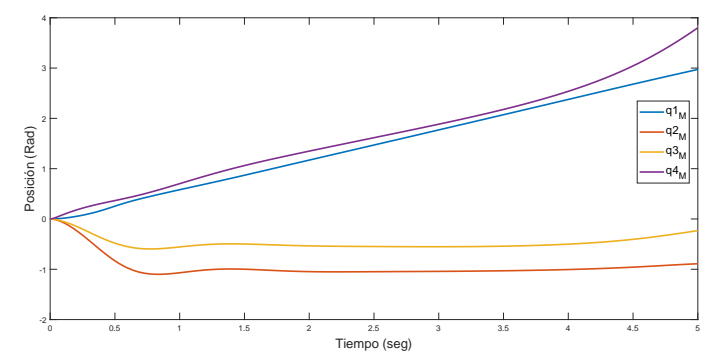

Figura 6: Evolución de las posiciones articulares del manipulador

\section{Validación}

El modelo dinámico obtenido está basado en las ecuaciones de Euler-Lagrange, por lo que para validarlo, se comparan sus resultados con los de un modelo obtenido mediante otro método. Para esto, se hace uso de la paquetería de robótica y visión artificial de Matlab diseñada por Peter Corke (Corke, 2017), la cual implementa un algoritmo recursivo basado en el método 
de Newton-Euler para evaluar la dinámica de robots manipuladores.

Al evaluar el modelo dinámico del manipulador obtenido a través de Matlab en el mismo intervalo de tiempo, con el mismo par de entrada $\tau_{0}$, se obtiene la evolución de las posiciones articulares del manipulador mostrada en la Figura 6, así, contrastando los resultados de ambas simulaciones, se observa que exhiben la misma evolución en las posiciones articulares del manipulador (ver Figura 7). Lo cual valida el modelo dinámico obtenido.

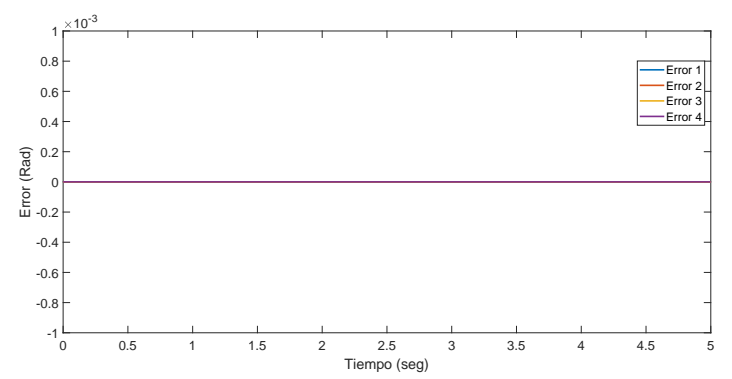

Figura 7: Error entre las posiciones calculadas por Euler-Lagrange y las calculadas por el método de Newton-Euler

\section{Conclusiones}

El cálculo del modelo cinemático del manipulador resulta indispensable para el estudio del movimiento del mismo en su espacio de trabajo, pues es con la matriz de transformación homogénea que se evalúa la posición alcanzada por el efector en el espacio cartesiano $[x, y, z]$ para todas las posiciones articulares $q$ alcanzables. Además, las matrices de rotación contenidas en las matrices de transformación homogénea de cada eslabón, son de especial utilidad para aplicar las transformaciones rígidas necesarias a los tensores de inercia de los eslabones durante el cálculo del modelo dinámico. El modelo dinámico calculado en este trabajo fue validado mediante su comparación con el modelo obtenido con la herramienta computacional MatlabSimulink, con lo cual se prueba que la metodología seguida en este artículo es válida y el modelo dinámico es confiable para el diseño de algoritmos de control basados en el modelo.

\section{English Summary}

\section{Kinematic and dynamic model validation of a four de- gree of freedom anthropomorphic robot manipulator.}

\section{Abstract}

In this paper, the kinematic and dynamic model of a 4 degree of freedom anthropomorphic robot manipulator are obtained by means of the Denavit - Hartenberg convention and the Euler - Lagrange formulation respectively. The kinematic model is used to construct the workspace of the robot manipulator. A software CAD -CAE is used to estimate the robot manipulator's physical parameters. The dynamic model is proven to be valid via its comparison with the model obtain through the use of a computational tool which uses the Newton-Euler algorithm.

Keywords:

Robot manipulator, Anthropomorphic robot, Kinematic model, Dynamic model, Euler-Lagrange.

\section{Agradecimientos}

Este trabajo ha sido realizado gracias al financiamiento de la SIP-IPN con número de registro 20194990 y 20195693.

\section{Referencias}

Antonio, C. J. M., 2012. Brazo robótico planar con dos grados de libertad. Ph.D. thesis, INSTITUTO POLITÉCNICO NACIONAL.

Arimoto, S., 1986. Stability and robustness of pd feedback control with gravity compensation for robot manipulator. Robotics: Theory and ApplicationsDSC 3, 67-72.

Chua, P. Y., Ilschner, T., Caldwell, D. G., 2003. Robotic manipulation of food products-a review. Industrial Robot: An International Journal 30 (4), 345354.

Corke, P., 2017. Robotics, vision and control: fundamental algorithms in MATLABR second, completely revised. Vol. 118. Springer.

Corke, P. I., 2007. A simple and systematic approach to assigning denavithartenberg parameters. IEEE transactions on robotics 23 (3), 590-594.

Flores, B. L. R., 2006. Cálculo de la cinemática y la dinámica del manipulador cinvestav-esime. Ph.D. thesis, INSTITUTO POLITECNICO NACIONAL.

Gautier, M., 1997. Dynamic identification of robots with power model. In: Proceedings of International Conference on Robotics and Automation. Vol. 3. IEEE, pp. 1922-1927.

Goldstein, H., Poole, C., Safko, J., 2002. Classical mechanics.

Kelly, R., Davila, V. S., Perez, J. A. L., 2006. Control of robot manipulators in joint space. Springer Science \& Business Media.

Markert, J., Merk, G., Oct. 20 2009. Manipulator with automatic control, especially for the food industry. US Patent 7,603,927.

Meike, D., Pellicciari, M., Berselli, G., 2013. Energy efficient use of multirobot production lines in the automotive industry: Detailed system modeling and optimization. IEEE Transactions on Automation Science and Engineering 11 (3), 798-809.

Merchán-Cruz, E., 2000. Metodología para la generación de trayectorias de manipuladores robóticos, su cinemática y su dinámica. Instituto Politécnico Nacional. México, DF.

Murray, R. M., 2017. A mathematical introduction to robotic manipulation. CRC press.

Ramírez-Gordillo, J., 2010. Planeación de trayectorias en sistemas de manipuladores robóticos múltiples. Ingeniería Mecánica, 328.

Shepherd, S., Buchstab, A., 2014. Kuka robots on-site. In: Robotic Fabrication in Architecture, Art and Design 2014. Springer, pp. 373-380.

Spong, M. W., Vidyasagar, M., 2008. Robot dynamics and control. John Wiley \& Sons.

\section{Apéndice A. Modelo dinámico del manipulador}

Apéndice A.1. Lagrangiano del manipulador

$$
\begin{aligned}
L(q, \dot{q}) & =\frac{1}{2} m_{1} l c_{1}^{2} \dot{q}_{1}^{2}+\frac{1}{2} I_{y y_{1}} \dot{q}_{1}^{2}+\frac{1}{2} m_{2} l c_{2}^{2}\left(c_{2}^{2} \dot{q}_{1}^{2}+\dot{q}_{2}^{2}\right) \\
& +\frac{1}{2}\left(\dot{q}_{1}^{2}\left(s_{2}^{2} I_{x x_{2}}+c_{2}^{2} I_{y y_{2}}\right)+\dot{q}_{2}^{2} I_{z z_{2}}\right)+\frac{1}{2} m_{3} l_{2}^{2} \dot{q}_{2}^{2} \\
& +2 l_{2} l c_{3}\left(\dot{q}_{2}^{2}+\dot{q}_{2} \dot{q}_{3}\right) c_{3}+l c_{3}^{2}\left(\dot{q}_{2}+\dot{q}_{3}\right)^{2}+\dot{q}_{1}{ }^{2}\left(l_{2}^{2} c_{2}^{2}\right.
\end{aligned}
$$




$$
\begin{aligned}
& \left.+2 l_{2} l c_{3} c_{2} c_{23}+l c_{3}^{2} c_{23}^{2}\right)+\frac{1}{2}\left(s_{2}^{2} \dot{q}_{1}^{2}\left(c_{3}^{2} T_{x x_{3}}+s_{3}^{2} I_{y y_{3}}\right)\right. \\
& +s_{2} c_{2} \dot{q}_{1}{ }^{2}\left(c_{3} s_{3} I_{x x_{3}}-c_{3} s_{3} I_{y y_{3}}\right)+c_{2}^{2} \dot{q}_{1}{ }^{2}\left(s_{3}^{2} T_{x x_{3}}+c_{3}^{2} I_{y y_{3}}\right) \\
& \left.+I_{z z_{3}}\left(\dot{q}_{2}+\dot{q}_{3}\right)^{2}\right)+\frac{1}{2} m_{4} l_{2}^{2} \dot{q}_{2}{ }^{2}+l_{3}^{2}\left(\dot{q}_{2}{ }^{2}+2 \dot{q}_{2} \dot{q}_{3}+\dot{q}_{3}{ }^{2}\right) \\
& +l c_{4}^{2}\left(\dot{q}_{2}^{2}+\dot{q}_{3}^{2}+\dot{q}_{4}{ }^{2}+2 \dot{q}_{2} \dot{q}_{3}+2 \dot{q}_{2} \dot{q}_{4}+2 \dot{q}_{3} \dot{q}_{4}\right)
\end{aligned}
$$

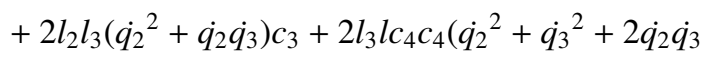

$$
\begin{aligned}
& \left.+\dot{q}_{2} \dot{q}_{3}+\dot{q}_{3} \dot{q}_{4}\right)+2 l_{2} l c_{4}\left(\dot{q}_{2}^{2}+\dot{q}_{2} \dot{q}_{3}+\dot{q}_{3} \dot{q}_{4}\right) c_{34} \\
& +l_{2}^{2} \dot{q}_{1}^{2} c_{2}^{2}+l_{3}^{2} \dot{q}_{1}^{2} c_{23}^{2}+l c_{4}^{2} \dot{q}_{1}^{2} c_{234}^{2} 2 l_{2} l_{3} \dot{q}_{1}^{2} c_{2} c_{23} \\
& +2 l_{3} l c_{4} \dot{q}_{1}^{2} c_{23} c_{234}+2 l_{2} l c_{4} \dot{q}_{1}^{2} c_{2} c_{234}+\frac{1}{2}\left(\left(s_{2}^{2} c_{3}^{2} c_{4}^{2} I_{x x_{4}}\right.\right. \\
& +s_{2}^{2} c_{3}^{2} s_{4}^{2} I_{y y_{4}}+2 s_{2} c_{2} s_{3} c_{3} c_{4}^{2} I_{x x_{4}}+2 s_{2} c_{2} s_{3} c_{3} s_{4}^{2} I_{y y_{4}} \\
& \left.+s_{3}^{2} c_{2}^{2} c_{4}^{2} I_{x x_{4}}+s_{3}^{2} c_{2}^{2} c_{4}^{2} I_{x x_{4}}+s_{3}^{2} c_{2}^{2} s_{4}^{2} I_{y y_{4}}\right) \dot{q}_{1}^{2}+\left(\left(2 \left(c_{2}^{2}\right.\right.\right. \\
& \left.\left.\left.-s_{2}^{2}\right) s_{3} c_{3}+2\left(c_{3}^{2}-s_{3}^{2}\right) s_{2} c_{2}\right)\left(s_{4} c_{4} I_{x x_{4}}-s_{4} c_{4} I_{y y_{4}}\right)\right) \dot{q}_{1}{ }^{2} \\
& +\left(s_{3}^{2} s_{2}^{2} s_{4}^{4} I_{x x_{4}} s_{3}^{2} s_{2}^{2} c_{4}^{2} I_{y y_{4}}-2 s_{3} c_{3} s_{2} c_{2} s_{4}^{2} I_{x x_{4}}\right. \\
& \left.-2 s_{3} c_{3} s_{2} c_{2} c_{4}^{2} I_{y y_{4}}+c_{3}^{2} c_{2}^{2} s_{4}^{2} I_{x x_{4}}+c_{3}^{2} c_{2}^{2} c_{4}^{2} I_{y y_{4}}\right) \dot{q}_{1}^{2}+\left(\dot{q}_{2}^{2}\right. \\
& \left.\left.+\dot{q}_{3}^{2}+\dot{q}_{4}^{2}+2 \dot{q}_{2} \dot{q}_{3}+2 \dot{q}_{2} \dot{q}_{4}+2 \dot{q}_{3} \dot{q}_{4}\right) I_{z z_{4}}\right)-l c_{1} m_{1} g \\
& -\left(l_{1}+l c_{2} s_{2}\right) m_{2} g-\left(l_{1}+l_{2} s_{2}+l c_{3} s_{23}\right) m_{3} g-\left(l_{1}+l_{2} s_{2}\right. \\
& \left.+l_{3} s_{2+3}+l c_{4} s_{234}\right) m_{4} g
\end{aligned}
$$

Apéndice A.1.1. Matriz de Inercia

$$
\mathbf{M}(\mathbf{q})=\left[\begin{array}{llll}
M_{11} & M_{12} & M_{13} & M_{14} \\
M_{21} & M_{22} & M_{23} & M_{24} \\
M_{31} & M_{32} & M_{33} & M_{34} \\
M_{41} & M_{42} & M_{43} & M_{44}
\end{array}\right]
$$

$$
\begin{aligned}
M_{11} & =m_{2} l c_{2}^{2} c_{2}^{2}+m_{3} l_{2}^{2} c_{2}^{2}+2 m_{3} l_{2} l c_{3} c_{2} c_{23}+m_{3} l c_{3}^{2} c_{23}^{2}+m_{4}\left(l_{2}^{2} c_{2}^{2}\right. \\
& \left.+l_{3} c_{23}^{2}+l c_{4}^{2} c_{234}^{2}+2 l_{2} l_{3} c_{2} c_{23}+2 l_{3} l c_{4} c_{23} c_{234}+2 l_{2} l c_{4} c_{2} c_{234}\right) \\
& +I_{y y_{1}}+s_{2}^{2} I_{x x_{1}}+c_{2}^{2} I_{y y_{2}}+I_{x x_{3}}\left(c_{3}^{2} s_{2}^{2}+s_{3}^{2} c_{2}^{2}\right. \\
& \left.-\frac{1}{2} \operatorname{sen}\left(2 q_{2}\right) \operatorname{sen}\left(2 q_{3}\right)\right)+I_{y y_{3}}\left(s_{3}^{2} s_{2}^{2}+c_{3} 1^{2} c_{2}^{2}\right. \\
& \left.-\frac{1}{2} \operatorname{sen}\left(2 q_{2}\right) \operatorname{sen}\left(2 q_{3}\right)\right)+\left(c_{4}^{2} I_{x x_{4}}+s_{4}^{2} I_{y y_{4}}\right)\left(s_{2}^{2} c_{3}^{2}+\frac{1}{2} \operatorname{sen}\left(2 q_{2}\right)\right. \\
& \left.+\operatorname{sen}\left(2 q_{3}\right)+s_{3}^{2} c_{2}^{2}\right)+\left(I_{x x_{4}}\right. \\
& \left.-I_{y y_{4}}\right)\left(\frac{1}{2} \cos \left(2 q_{2}\right) \operatorname{sen}\left(2 q_{3}\right) \operatorname{sen}\left(2 q_{4}\right)\right. \\
& \left.+\frac{1}{2} \cos \left(2 q_{3}\right) \operatorname{sen}\left(2 q_{2}\right) \operatorname{sen}\left(2 q_{1}\right)\right)+\left(s_{4}^{2} I_{x x_{4}}-c_{4}^{2} I_{y y_{4}}\right)\left(s_{2}^{2} s_{3}^{2}\right. \\
& \left.-\frac{1}{2} \operatorname{sen}\left(2 q_{2}\right) \operatorname{sen}\left(2 q_{3}\right)+c_{2}^{2} c_{3}^{2}\right) \\
M_{12}= & M_{13}=M_{14}=M_{21}=M_{31}=M_{41}=0 \\
M_{22}= & m_{2} l c_{2}^{2}+m_{3} l_{2}^{2}+2 m_{3} l_{2} l c_{3} c_{3}+m_{3} l c_{3}^{2}+m_{4}\left(l_{2}^{2}+l_{3}^{2}+l c_{4}^{2}\right.
\end{aligned}
$$

$$
\begin{aligned}
& \left.+l_{2} l_{3} c_{3}+2 l_{3} l c_{4} c_{4}+2 l_{2} l c_{4} c_{34}\right)+I_{z z_{2}}+I_{z z 3} \\
M_{23} & =m_{3} l_{2} l c_{3} c_{3}+m_{3} l c_{3}^{2}+m_{4}\left(l_{3}^{2}+l c_{4}^{2}+l_{2} l_{3} c_{3}\right. \\
& \left.+2 l_{3} l c_{4} c_{4}+l_{2} l c_{4} c_{34}\right)+I_{z z_{3}} \\
M_{24} & =m_{4}\left(l c_{4}^{2}+l_{3} l c_{4} c_{4}+l_{2} l c_{4} c_{34}\right) \\
M_{32} & =m_{3} l_{2} l c_{3} c_{3}+m_{3} l c_{3}^{2}+m_{4}\left(l_{3}^{2}+l c_{4}^{2}+2 l_{2} l_{3} c_{3}+2 l_{3} l c_{4} c_{4}\right. \\
& \left.+l_{3} l c_{4} c_{34}\right)+I_{z z 3} \\
M_{33} & =m_{3} l c_{3}^{2}+m_{4}\left(l_{3}^{2}+l c_{4}^{2}+2 l_{3} l c_{4} c_{4}\right)+I_{z z_{3}} \\
M_{34} & =m_{4}\left(l c_{4}^{2}+l_{3} l c_{4} c_{4}\right) \\
M_{42} & =m_{4}\left(l c_{4}^{2}+l_{3} l c_{4} c_{4}+l_{2} l c_{4} c_{34}\right) \\
M_{43} & =m_{4}\left(l c_{4}^{2}+l_{3} l c_{4} c_{4}\right) \\
M_{44} & =m_{4} l c_{4}^{2}
\end{aligned}
$$

Apéndice A.1.2. Matriz de Coriolis

$$
\mathbf{C}(\mathbf{q}, \dot{\mathbf{q}})=\left[\begin{array}{llll}
C_{11} & C_{12} & C_{13} & C_{14} \\
C_{21} & C_{22} & C_{23} & C_{24} \\
C_{31} & C_{32} & C_{33} & C_{34} \\
C_{41} & C_{42} & C_{43} & C_{44}
\end{array}\right]
$$

$C_{11}=2 m_{2} l c_{2}^{2} c_{2} s_{2} \dot{q}_{2}+\left(\left(I_{x x_{2}}-I_{y y_{2}}\right) \operatorname{sen}\left(2 q_{2}\right)+\left(I_{x x_{3}}\right.\right.$

$\left.-I_{y y_{3}}\right) \operatorname{sen}\left(2 q_{2}\right) \cos \left(2 q_{3}\right)+\left(I_{x x_{3}}-I_{y y_{3}}\right) \operatorname{sen}\left(2 q_{3}\right) \cos \left(2 q_{2}\right)$

$\left.-\left(2 m_{4}\right)\left(l_{2}^{2} c_{2} s_{2}+l_{2} l_{3} c_{23} s_{2}+l_{2} l c_{4} c_{234} s_{2}\right)\right) \dot{q}_{2}$

$C_{12}=\left((-2)\left(m_{3} l_{2}^{2} c_{2} s_{2}+m_{3} l_{2} l c_{3} c_{2} s_{23}+m_{3} l_{2} l c_{3} c_{23} s_{2}\right.\right.$

$\left.+m_{3} l c_{3}^{2} c_{23} s_{23}\right)-2 m_{4}\left(l_{3}^{2} c_{23} s_{23}+l c_{4} c_{234} s_{234}\right.$

$\left.+l_{2} l_{3} c_{2} s_{23}+l_{3} l c_{4} c_{23} s_{234}+l_{3} l_{4} c_{234} s_{23}+l_{2} l c_{4} c_{2} s_{234}\right)$

$+\left(c_{4}^{2} I_{x x_{4}}+s_{4}^{2} I_{y y_{4}}\right)\left(c_{3}^{2} \operatorname{sen}\left(2 q_{2}\right)-s_{3}^{2} \operatorname{sen}\left(2 q_{2}\right)\right.$

$\left.+\operatorname{sen}\left(2 q_{3}\right) \cos \left(2 q_{2}\right)\right)+\left(s_{4} i_{x x_{4}}+c_{4}^{2} I_{y y_{4}}\right)\left(s_{3}^{2} \operatorname{sen}\left(2 q_{2}\right)\right.$

$\left.-c_{3}^{2} \operatorname{sen}\left(2 q_{2}\right)-\operatorname{sen}\left(2 q_{3}\right) \cos \left(2 q_{2}\right)\right)+\left(I_{y y_{4}}\right.$

$\left.-I_{x x_{4}}\right)\left(\operatorname{sen}\left(2 q_{3}\right) \operatorname{sen}\left(2 q_{4}\right) \operatorname{sen}\left(2 q_{2}\right)\right)+\left(I_{x x_{4}}\right.$

$\left.\left.-I_{y y_{4}}\right)\left(\cos \left(2 q_{3}\right) \operatorname{sen}\left(2 q_{1}\right) \cos \left(2 q_{2}\right)\right)\right) \dot{q}_{1}$

$C_{13}=\left(2 m_{3} l_{2} l c_{3} c_{2} s_{23}+2 m_{3} l c_{3}^{2} c_{23} s_{23}+\left(I_{x x_{3}}\right.\right.$

$\left.-I_{y y_{3}}\right) \cos \left(2 q_{2}\right) \operatorname{sen}\left(2 q_{3}\right)+\left(I_{x x_{3}}-I_{y y_{3}}\right) \operatorname{sen}\left(2 q_{2}\right) \cos \left(2 q_{3}\right)$

$-2 m_{4}\left(l_{3}^{2} c_{23} s_{23}+l c_{4} c_{234} s_{234}+l_{2} l_{3} c_{2} s_{23}+l_{3} l_{4} c_{23} s_{234}\right.$

$\left.+l_{3} l c_{4} c_{234} s_{23}+l_{2} l c_{4} c_{2} s_{234}\right)+\left(c_{4}^{2} I_{x x_{4}}\right.$

$\left.+s_{4}^{2} I_{y y_{4}}\right)\left(-s_{2}^{2} \operatorname{sen}\left(2 q_{3}\right)+c_{2}^{2} \operatorname{sen}\left(2 q_{3}\right)+\operatorname{sen}\left(2 q_{2}\right) \cos \left(2 q_{3}\right)\right)$

$+\left(s_{4}^{2} I_{x x_{4}}+c_{4}^{2} I_{y y_{4}}\right)\left(s_{2}^{2} \operatorname{sen}\left(2 q_{3}\right)-c_{3} \operatorname{sen}\left(2 q_{3}\right)\right.$

$\left.-\operatorname{sen}\left(2 q_{2}\right) \cos \left(2 q_{3}\right)\right)+\left(I_{x x_{4}}\right.$

$\left.-I_{y y_{4}}\right)\left(\cos \left(2 q_{2}\right) \operatorname{sen}\left(2 q_{4}\right) \cos \left(2 q_{3}\right)\right.$

$\left.\left.-\operatorname{sen}\left(2 q_{2}\right) \operatorname{sen}\left(2 q_{4}\right) \operatorname{sen}\left(2 q_{3}\right)\right)\right) \dot{q}_{1}$

$C_{14}=\dot{q}_{1}\left(-2 m_{4}\left(l c_{4}^{2} c_{234} s_{234}+l_{3} l c_{4} c_{23} s_{234}+l_{2} l c_{4} c_{2} s_{234}\right)\right.$

$+\left(I_{y y_{4}}-I_{x x_{4}}\right)\left(s_{2}^{2} c_{2}^{2} \operatorname{sen}\left(2 q_{4}\right)+s_{3}^{2} c_{2}^{2} \operatorname{sen}\left(2 q_{4}\right)\right.$ 
$\left.+\frac{1}{2} \operatorname{sen}\left(2 q_{2}\right) \operatorname{sen}\left(2 q_{3}\right) \operatorname{sen}\left(2 q_{4}\right)\right)+\left(I_{x x_{4}}\right.$

$\left.-I_{y y_{4}}\right)\left(s_{3}^{2} s_{2}^{2} \operatorname{sen}\left(2 q_{4}\right)+c_{2}^{2} c_{3}^{2} \operatorname{sen}\left(2 q_{4}\right)\right.$

$+\cos \left(2 q_{2}\right) \operatorname{sen}\left(2 q_{3}\right) \cos \left(2 q_{4}\right)$

$\left.\left.+\cos \left(2 q_{3}\right) \operatorname{sen}\left(2 q_{2}\right) \cos \left(2 q_{4}\right)\right)\right)$

$C_{21}=\dot{q}_{1}\left(m_{2} l c_{2}^{2} c_{2} s_{2} \dot{q}_{2}+m_{3} l_{2}^{2} c_{2} s_{2} \dot{q}_{2}+m_{3} l_{2} l c_{3} c_{2} s_{23}\right.$

$+m_{3} l_{2} l c_{3} c_{23} s_{2}+m_{3} l c_{3}^{2} c_{23} s_{23}+m_{4}\left(l_{2}^{2} c_{2} s_{2}+l_{3}^{2} c_{23} s_{23}\right.$

$+l c_{4} c_{234} s_{234}+l_{2} l_{3} c_{2} s_{23}+l_{2} l_{3} c_{23} s_{2}+l_{3} l_{c_{4}} c_{23} s_{234}$

$\left.+l_{3} l c_{4} c_{234} s_{23}+l_{2} l c_{4} c_{2} s_{234}+l_{2} l c_{4} c_{234} s_{2}\right)-\frac{1}{2}\left(\left(I_{x x_{2}}\right.\right.$

$\left.-I_{y y_{2}}\right) \operatorname{sen}\left(2 q_{2}\right)+\left(I_{x x_{3}}-I_{y y_{3}}\right) \cos \left(2 q_{3}\right) \operatorname{sen}\left(2 q_{2}\right)$

$+\left(I_{x x_{3}}-I_{y y_{3}}\right) \operatorname{sen}\left(2 q_{3}\right) \cos \left(2 q_{2}\right)+\left(I_{x x_{4}}\right.$

$\left.-I_{y y_{4}}\right)\left(\cos \left(2 q_{3}\right) \operatorname{sen}\left(2 q_{2}\right) \cos \left(2 q_{4}\right)\right.$

$+\cos \left(2 q_{4}\right) \operatorname{sen}\left(2 q_{3}\right) \cos \left(2 q_{2}\right)$

$\left.\left.\left.+\operatorname{sen}\left(2 q_{4}\right)\left(\cos \left(2 q_{3}\right) \cos \left(2 q_{2}\right)-\operatorname{sen}\left(2 q_{3}\right) \operatorname{sen}\left(2 q_{2}\right)\right)\right)\right)\right)$

$C_{22}=-2 m_{3} l_{2} l c_{3} s_{3} \dot{q}_{3}-2 m_{4}\left(\dot{q}_{3}\left(l_{2} l_{3} s_{3}+l_{2} l c_{4} s_{34}\right)\right.$

$\left.+\dot{q}_{4}\left(l_{3} l c_{4} s_{4}+l_{2} l c_{4} s_{34}\right)\right)$

$C_{23}=\dot{q}_{3}\left(-m_{3} l_{2} l c_{3} s_{3}\right)-m_{4}\left(\dot{q}_{3}\left(l_{2} l_{3} s_{3}+l_{2} l c_{4} s_{34}\right)\right.$

$\left.+\dot{q}_{4}\left(2 l_{3} l c_{4} s_{4}+l_{2} l c_{4} s_{34}\right)\right)$

$C_{24}=-m_{4}\left(l_{2} l c_{4} s_{34} \dot{q}_{3}+\dot{q}_{4}\left(l_{3} l c_{4} s_{4}+l_{2} l c_{4} s_{34}\right)\right)$

$C_{31}=\dot{q}_{1}\left(m_{3} l_{2} l c_{3} c_{2} s_{23}+m_{3} l c_{3}^{2} c_{23} s_{23} m_{4}\left(l_{3}^{2} c_{23} s_{23}\right.\right.$

$+l c_{4} c_{234} s_{234}+l_{2} l_{3} c_{2} s_{23}+l_{3} l c_{4} c_{23} s_{234} l_{3} l c_{4} c_{234} s_{23}$

$\left.+l_{2} l c_{4} c_{2} s_{234}\right)-\frac{1}{2}\left(\left(I_{y y_{3}}+I_{x x_{3}}\right) \cos \left(2 q_{2}\right) \operatorname{sen}\left(2 q_{3}\right)\right.$

$+\left(I_{x x_{3}}-I_{y y_{3}}\right) \operatorname{sen}\left(2 q_{2}\right) \cos \left(2 q_{3}\right)\left(I_{x x_{4}}\right.$

$\left.-I_{y y_{4}}\right)\left(\cos \left(2 q_{2}\right) \operatorname{sen}\left(2 q_{3}\right) \cos \left(2 q_{4}\right)\right.$

$+\cos \left(2 q_{4}\right) \operatorname{sen}\left(2 q_{2}\right) \cos \left(2 q_{3}\right)$

$\left.\left.\left.+\operatorname{sen}\left(2 q_{4}\right)\left(\cos \left(2 q_{2}\right) \cos \left(2 q_{3}\right)-\operatorname{sen}\left(2 q_{2}\right) \operatorname{sen}\left(2 q_{3}\right)\right)\right)\right)\right)$

$C_{32}=\dot{q}_{2}\left(m_{3} l_{2} l c_{3} s_{3}+m_{4}\left(l_{2} l_{3} s_{3}+l_{2} l c_{4} s_{34}\right)\right)$

$C_{33}=-2 m_{4} l_{3} l c_{4} s_{4} \dot{q}_{4}$

$C_{34}=-2 m_{4}\left(\dot{q}_{2}\left(2 l_{3} l c_{4} s_{4}\right)+\dot{q}_{4}\left(l_{3} l c_{4} s_{4}\right)\right)$

$C_{41}=-\frac{1}{2} \dot{q}_{1}\left(\left(\cos \left(2 q_{3}\right) \cos \left(2 q_{2}\right) \operatorname{sen}\left(2 q_{4}\right)\right.\right.$

$-\operatorname{sen}\left(2 q_{2}\right) \operatorname{sen}\left(2 q_{3}\right) \operatorname{sen}\left(2 q_{4}\right)$

$+\cos \left(2 q_{4}\right)\left(\cos \left(2 q_{2}\right) \operatorname{sen}\left(2 q_{3}\right)\right.$

$\left.\left.\left.+\cos \left(2 q_{3}\right) \operatorname{sen}\left(2 q_{2}\right)\right)\right)\left(I_{x x_{4}}-I_{y y_{4}}\right)\right)$

$C_{42}=m_{4}\left(\dot{q}_{2}\left(l_{3} l c_{4} s_{4}+l_{2} l c_{4} s_{34}\right)\right)$

$C_{43}=+m_{4}\left[\dot{q}_{2}\left(2 l_{3} l c_{4} s_{4}\right)+l_{3} l c_{4} s_{4} \dot{q}_{3}\right]$

$C_{44}=0$

Apéndice A.1.3. Vector de Gravedad

$$
\mathbf{G}(\mathbf{q})=\left[\begin{array}{llll}
G_{1} & G_{2} & G_{3} & G_{4}
\end{array}\right]^{T}
$$

donde

$$
\begin{aligned}
& I_{1}=\left[I_{x x 1}, I_{y y 1}, I_{z z 1}\right]=[2956,2711,2521] * 10^{-10} \\
& I_{2}=\left[I_{x x 2}, I_{y y 2}, I_{z z 2}\right]=[6214,20224,14473] * 10^{-10}
\end{aligned}
$$

$$
\begin{aligned}
& G_{1}=0 \\
& G_{2}=c_{2} m_{2} c_{2}-l_{2} m_{3} c_{2}-l c_{3} m_{3} c_{23}+m_{4}\left(l_{2} c_{2}+l_{3} c_{23}+l c_{4} c_{234}\right) \\
& G_{3}=l c_{3} m_{3} c_{23}+m_{4}\left(l_{3} c_{23}+l c_{4} c_{234}\right) \\
& G_{4}=m_{4} l c_{4} c_{234}
\end{aligned}
$$

\section{Apéndice B. Tabla de parámetros del manipulador}

\begin{tabular}{|l|c|c|c|}
\hline Parámetro & Notación & Valor & Unidades \\
\hline $\begin{array}{l}\text { Longitud } \\
\text { eslabón 1 }\end{array}$ & $L_{1}$ & 0.030 & $\mathrm{~m}$ \\
\hline $\begin{array}{l}\text { Longitud } \\
\text { eslabón 2 }\end{array}$ & $L_{2}$ & 0.124 & $\mathrm{~m}$ \\
\hline $\begin{array}{l}\text { Longitud } \\
\text { eslabón 3 }\end{array}$ & $L_{3}$ & 0.128 & $\mathrm{~m}$ \\
\hline $\begin{array}{l}\text { Longitud } \\
\text { eslabón 4 }\end{array}$ & $L_{4}$ & 0.160 & $\mathrm{~m}$ \\
\hline $\begin{array}{l}\text { Centro de masa } \\
\text { eslabón 1 }\end{array}$ & $l_{c 1}$ & -0.013 & $\mathrm{~m}$ \\
\hline $\begin{array}{l}\text { Centro de masa } \\
\text { eslabón 2 }\end{array}$ & $l_{c 2}$ & -0.056 & $\mathrm{~m}$ \\
\hline $\begin{array}{l}\text { Centro de masa } \\
\text { eslabón 3 }\end{array}$ & $l_{c 3}$ & -0.075 & $\mathrm{~m}$ \\
\hline $\begin{array}{l}\text { Centro de masa } \\
\text { eslabón 4 }\end{array}$ & $l_{c 4}$ & -0.104 & $\mathrm{~m}$ \\
\hline Masa eslabón 1 & $m_{1}$ & 0.069 & $\mathrm{~kg}$ \\
\hline Masa eslabón 2 & $m_{2}$ & 0.044 & $\mathrm{~kg}$ \\
\hline Masa eslabón 3 & $m_{3}$ & 0.097 & $\mathrm{~kg}$ \\
\hline Masa eslabón 4 & $m_{4}$ & 0.203 & $\mathrm{~kg}$ \\
\hline $\begin{array}{l}\text { Tensor de inercia } \\
\text { eslabón 1 }\end{array}$ & $I_{1}$ & $\operatorname{Diag}\left(I_{1}\right)$ & $\mathrm{kg} \cdot \mathrm{m}^{2}$ \\
\hline $\begin{array}{l}\text { Tensor de inercia } \\
\text { eslabón 2 }\end{array}$ & $I_{2}$ & $\operatorname{Diag}\left(I_{2}\right)$ & $\mathrm{kg} \cdot \mathrm{m}^{2}$ \\
\hline $\begin{array}{l}\text { Tensor de inercia } \\
\text { eslabón 3 }\end{array}$ & $I_{3}$ & $\operatorname{Diag}\left(I_{3}\right)$ & $\mathrm{kg} \cdot \mathrm{m}^{2}$ \\
\hline $\begin{array}{l}\text { Tensor de inercia } \\
\text { eslabón 4 }\end{array}$ & $I_{4}$ & $\operatorname{Diag}\left(I_{4}\right)$ & $\mathrm{kg} \cdot \mathrm{m}^{2}$ \\
\hline $\begin{array}{l}\text { Coeficientes de } \\
\text { fricción viscosa }\end{array}$ & $\mathrm{B}$ & $\operatorname{Diag}(B)$ & $\frac{\mathrm{kg} \cdot \mathrm{m}^{2}}{\mathrm{~s}}$ \\
\hline
\end{tabular}

Tabla B.2: Tabla de parametros del robot manipulador

$$
\begin{gathered}
I_{3}=\left[I_{x x 3}, I_{y y 3}, I_{z z 3}\right]=[11963,68870,58373] * 10^{-10} \\
I_{4}=\left[I_{x x 4}, I_{y y 4}, I_{z z 4}\right]=[15828,257728,253343] * 10^{-10} \\
{\left[B_{1}, B_{2}, B_{3}, B_{4}\right]=[0,1668,0,1668,0,1668,0,1668]}
\end{gathered}
$$

